\title{
Conducting ethical clinical trials for L. donovani in the Bihar region of India
}

This article was published in the following Dove Press journal:

Open Access Journal of Clinical Trials

2 August 2010

Number of times this article has been viewed

\section{Tiffany Crawford \\ Brian Vesely}

Global Health, University of South Florida, Tampa, FL, USA
Correspondence: Brian Vesely Global Health, University of South Florida, 3720 Spectrum Blvd, Suite 304,Tampa, FL 33612, USA

$\mathrm{Tel}+$ I 8139275994

$\mathrm{Fax}+$ I 8139740992

Email bvesely@health.usf.edu

\begin{abstract}
Visceral leishmaniasis is a severe disease that disproportionally afflicts the Bihar state in India with $40 \%$ of the worldwide disease burden. Drug resistance, poor adherence, extreme poverty, malnutrition and certain living conditions have made control and treatment of the disease difficult. There is a great need for new drugs and control programs to reduce the disease burden of this debilitating and potentially fatal disease. Drug discovery research on leishmaniasis is being conducted in the USA and this raises issues concerning the ethical conduct of international clinical trials. Ethical principles dictate that the Bihari who need these drugs should be included in clinical trials. Additional safeguards for the ethical conduct of clinical trials on developing countries by developed countries have been formulated elsewhere. These include collaborative partnership, social value, scientific validity, fair selection of study population, favorable risk-benefit ratio, independent review, informed consent, and respect for recruited participants and study communities. These principles are applied to the Bihari context, and issues of ancillary care and post-trial access are also addressed. The socio-cultural context of the region is discussed in order to give researchers the tools to obtain meaningful informed consent. A description of the Bihari context and relevant ethical considerations should facilitate the design and conduct of ethical clinical trials for L. donovani.
\end{abstract}

Keywords: L. donovani, ethics, clinical trials, Bihar

\section{Introduction}

Leishmania donovani is a flagellated protozoan parasite of animals and humans responsible for the presentation of leishmaniasis. L. donovani has a divalent life cycle consisting of a promastigote form inside a sand fly vector and an amastigote form that lives in the parasitopherous vacuole of macrophages in humans. L. donovani causes the visceral form of leishmaniasis that is characterized by hepatomegaly and splenomegaly and results in mortality when untreated. ${ }^{1}$ 90\% of cases occur in Bangladesh, India, Nepal, Sudan, and Brazil. ${ }^{2}$ Visceral leishmaniasis is also endemic in Ethiopia. ${ }^{3}$ There are 500,000 new cases reported each year, and 200,000 of those cases come from the Bihar state in India. ${ }^{4}$ Bihar comprises $40 \%$ of the worldwide disease burden of visceral leishmaniasis and warrants international attention. $65 \%$ of cases $(130,000)$ in Bihar are resistant to pentavalent antimonials, the current first line drug treatment of leishmaniasis worldwide. ${ }^{5}$ Insufficient treatment schemes and poor adherence in the region have led to an increasing unresponsiveness to the pentavalent antimonials. ${ }^{6}$ Widespread resistance in the region makes control of the disease more difficult and raises ethical questions about prescribing a drug when it is becoming increasingly resistant. New drugs and control programs are needed to reduce the disease burden of this debilitating and potentially fatal disease. 
Bihar is currently the world "hot spot" for visceral leishmaniasis for several reasons. Drug resistance, poor adherence, extreme poverty, malnutrition and certain living conditions have made control and treatment of the disease difficult. Resistance to available treatment should be addressed by clinical trials of promising drugs. Researchers and medical personnel need to evaluate the reasons behind poor treatment adherence and incorporate these findings into future treatment protocols. Clinical trials of promising treatments for leishmaniasis are needed in Bihar. Drug discovery research on leishmaniasis is being conducted in the USA, and this raises issues concerning the ethical conduct of international clinical trials. Ethical clinical trials that take into consideration the cultural, economic, and social issues of the region need to be conducted.

\section{Ethical issues in international clinical trials}

Autonomy, beneficence and justice were identified as key ethical principles in research involving human participants in the Belmont Report by the National Commission for the Protection of Human Subjects of Biomedical and Behavioral Research in 1979. ${ }^{7}$ These guidelines were established following ethical concerns that arose in the Syphilis Study at Tuskegee, Alabama. ${ }^{7}$ According to this and other documents, potential participants are to be viewed as autonomous agents who have the right to self-determination. ${ }^{7,8}$ An autonomous person is able to make decisions singularly based on evaluating the potential risks and benefits of participating in a research study and relating those risks and benefits to personal goals and values. ${ }^{7}$ Informed consent is a key tool in ensuring a person's autonomy as it allows an individual the opportunity to decline participation. Beneficence is based on several requirements, including an obligation to help others, to do no harm, and to maximize the potential benefits and minimize possible harms of a research study. ${ }^{7}$ The riskto-benefit ratio is a key concept in evaluating beneficence. Justice requires that individuals and groups be treated fairly and equitably in terms of bearing the burdens and receiving the benefits of research. ${ }^{7}$ Justice is an equitable balance of the potential risks and benefits within and across communities. The inclusion/exclusion criteria are important indicators of justice in any research study.

These ethical principles are embodied in several US documents, including the National Institute of Health (NIH) Protecting Human Research Participants. ${ }^{7}$ Based on these principles, ethical standards for clinical trials in the US include review and approval of study protocol by an ethics review board, minimization of the potential risks, an acceptable risk-to-benefit ratio and a meaningful informed consent process. ${ }^{9}$ Questions arise concerning the applicability of these ethical standards and procedures in US-sponsored clinical trials conducted in developing countries and whether such trials require different or additional ethical considerations. ${ }^{9}$ Achieving these ethical standards in developing countries where resources are limited and the health infrastructure is ill-equipped may require additional steps and safeguards.

The ethical debate of research in developing countries is focused on the standard of care, post-trial benefits, and issues of informed consent. ${ }^{10}$ Whether a control group in a clinical trial in a developing country should receive the same treatment as it would in a developed country remains controversial. ${ }^{9}$ The position of the US National Bioethics Advisory Commission is that "a placebo control, or any other control that is less effective than an established, effective treatment, is not ethically acceptable", 11 states that "at the conclusion of the study, patients entered into the study are entitled to be informed about the outcome of the study and to share any benefits that result from it, for example, access to interventions identified as beneficial in the study or to other appropriate care or benefits" ${ }^{11}$ However, the FDA officially abandoned the 2008 version of the Declaration of Helsinki (DOH), and the extent of obligation for US researchers to provide post-trial access remains unclear. ${ }^{10}$ The informed consent form (ICF) is used in the US to indicate meaningful participant consent and supports the principle of autonomy. However, research shows that many participants are unaware that they are in an experiment and strongly hold to the belief that they are receiving the best proven treatment despite signing a document that states otherwise. ${ }^{12}$ The legitimacy of the signed ICF is further challenged when the form is translated to another language without simultaneous consideration of cultural factors. Moreover, a signed ICF may have little meaning in a culture in which patients defer to medical "experts" or in strongly hierarchal societies where deference is the norm, as occurs in India.

It is generally accepted that these substantive ethical principles (autonomy, beneficence, and justice) apply universally. They have been adopted internationally and incorporated into governmental policy statements including the Ethical Guidelines for Biomedical Research on Human Participants, Indian Council of Medical Research. ${ }^{13}$ However, the procedures used to apply these universal principles are necessarily context specific and depend on many social, cultural, and political factors. ${ }^{14}$ Debate over the application 
of accepted ethical principles is due to their interpretive and sometimes contradictory nature. ${ }^{10}$ The $\mathrm{DOH}$ and other similar documents are not meant to provide researchers with precise procedures for the conduct of ethical research; rather these documents provide abstract principles that must be thoughtfully applied to a specific context, a process that requires moral reasoning and interpretation. ${ }^{15}$ Scientists involved in international research must be knowledgeable of and sensitive to the social, economic, and political issues relevant to their participants and the ways in which these factors contribute to their health status. ${ }^{15}$

Additional ethical principles for the conduct of clinical trials in developing countries by developed countries have been formulated. These include collaborative partnership, social value, scientific validity, fair selection of study population, favorable risk-benefit ratio, independent review, informed consent, and respect for recruited participants and study communities. ${ }^{10} \mathrm{~A}$ description of the Bihar context and relevant ethical considerations should facilitate the design and conduct of ethical clinical trials for $L$. donovani.

\section{Bihar background and context}

Bihar is one of the poorest and most densely populated states in India ${ }^{16}$ with a population of approximately 82.9 million. ${ }^{17}$ The disease burden of visceral leishmaniasis is unequally distributed in the population. Men are diagnosed 4 times more often than women. ${ }^{16}$ This disparity is likely due to the fact that men have an increased risk of exposure to sand flies compared with women because they work outside more and sleep outside during the summer months. ${ }^{18}$ Also, women tend to wear more clothing, a fact that can help prevent sand fly bites. ${ }^{18}$ However, this statistic might be inaccurate because women are less likely to seek out treatment for infection than men. ${ }^{18}$ Individuals can be infected with leishmaniasis at any point, but immunity is usually conferred after the first infection. However, certain factors such as genetics, malnutrition, and immune status can interfere with acquired immunity. This phenomenon is part of the reason why only $33 \%$ of the cases in Bihar are in children aged $2-11 .{ }^{18}$ Given the high rate of infection, an effective and safe treatment for children is needed. Bihar has a high rate of malnutrition, which has important implications for leishmaniasis. ${ }^{18}$ Malnutrition impairs immunity to visceral leishmaniasis, so people can be infected multiple times. ${ }^{19}$ Malnourishment might be a reason why adults in the hyperendemic region are getting leishmaniasis when they should have conferred immunity at a young age.

There are many other factors that lead to increased prevalence of leishmaniasis in Bihar including the nature of the reservoir, educational challenges, and extreme poverty. Dogs are a reservoir of $L$. donovani parasites in India, and they are often asymptomatic when seropositive with leishmania. ${ }^{20}$ The lack of symptoms makes it difficult and expensive to know when it is appropriate to cull dogs that might be infected. About $80 \%$ of the population is illiterate, and $82 \%$ of the population makes less than Rs 9,660 (US\$185) per year. ${ }^{18}$ According to Parker and Kozel, "Indian poverty is linked to economic, social, cultural, and political factors that interact to maintain long-term structural disparities in opportunities and resources". ${ }^{21}$ Extreme poverty makes it difficult to prevent, treat, and control visceral leishmaniasis. To prevent infection with leishmaniasis, sand fly bed nets and insecticide repellant are needed, but it is difficult for the poor to afford these items. In addition, illiteracy makes educating potential users more difficult. The current popular treatment in Bihar is the use of pentavalent antimonials, but $65 \%$ of cases in Bihar are now resistant to that drug. ${ }^{22}$ Other drugs that could be effective in treating leishmaniasis, such as miltefosine and amphotericin B are too expensive for the majority of Biharis. See Figure 1 for a summary of treatment costs. Liposomal amphotericin B is a relatively new drug proven effective in Bihar and the cost of treatment is US\$148.22 (Rs 6684.7) per patient. ${ }^{23}$ It is a single dose regimen, which facilitates easier delivery than standard amphotericin B (15 infusions over 30 days) and avoids issues of adherence. However, with an annual total healthcare expenditure of US\$35 (Rs 1586) per capita, it is still financially inaccessible to the majority of the population. ${ }^{24}$

Approximately $91 \%$ of the visceral leishmaniasis cases in Bihar occur in rural settings..$^{25}$ The people in rural Bihar live in mud houses and are surrounded by animals and vegetation that facilitate sand fly breeding. ${ }^{18}$ The remoteness of the problem makes it more difficult to enact prevention measures than in a city with more resources. The low castes' households in Bihar are predominantly located in rural areas. ${ }^{21}$ Poverty in these areas includes structural poverty perpetuated by the caste system and continues generationto-generation. ${ }^{18}$ Poverty is exacerbated by visceral leishmaniasis, because support from the government is limited and infected Biharis often have to sell their land and possessions to pay for treatment. ${ }^{18}$

Caste is a known determinant of poverty in India, ${ }^{21}$ and the burden of disease for visceral leishmaniasis is disproportionally represented by the poorest. ${ }^{25}$ The Indian caste system is a social stratification based on heredity called the Chaturvarna system, and structures social groups among Hindus. ${ }^{18}$ The Scheduled Castes (also called Dalits) and Schedules Tribal 


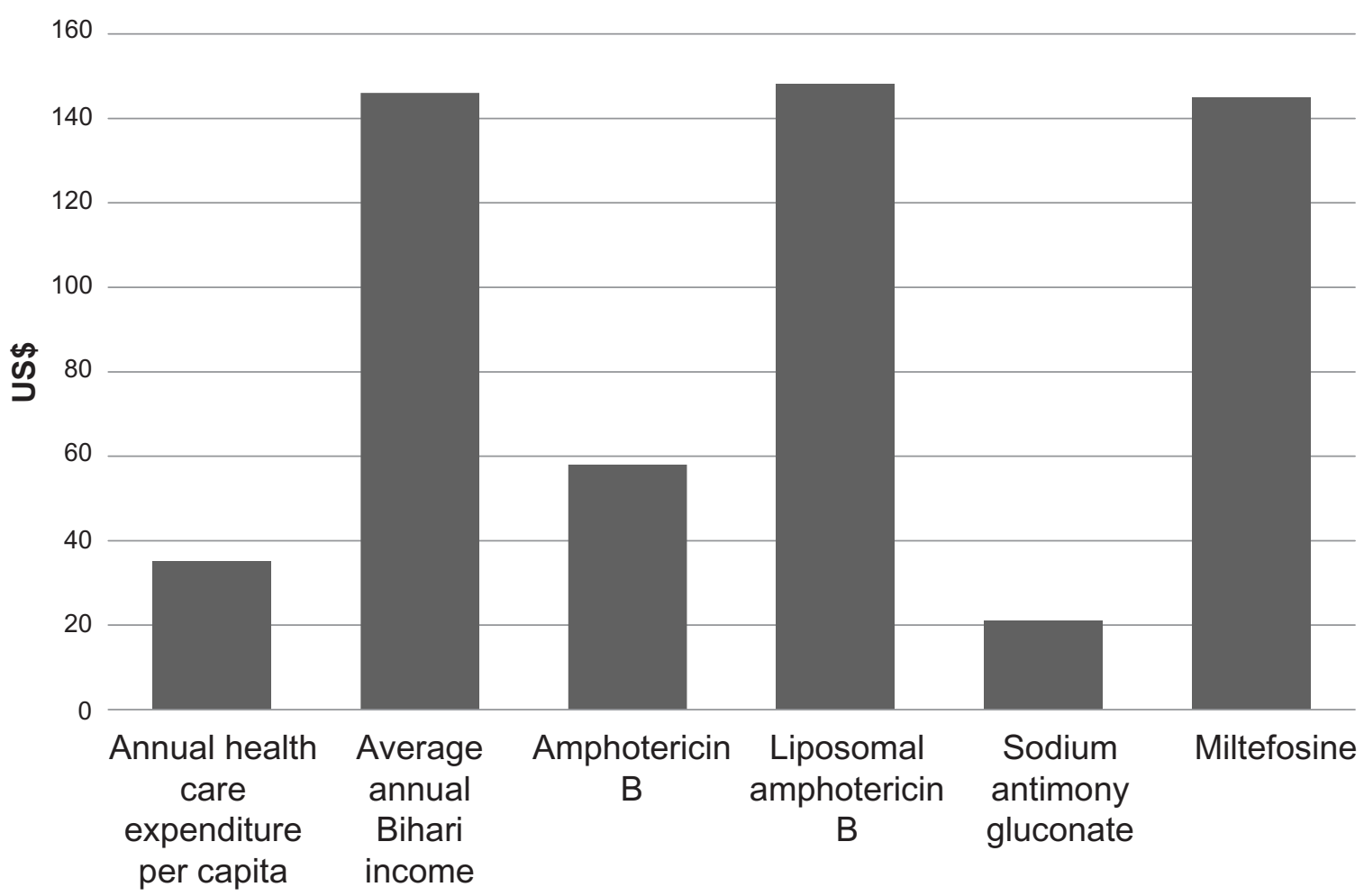

Figure I Summary of treatment cost for VL and Bihari financial status.

communities (also called Adivasis) were historically excluded from the Chaturvarna system and Indian society as a whole. ${ }^{18}$ Traditionally, they are labor workers with no potential for social mobility and are considered the most disadvantaged groups in the country. ${ }^{18}$ Almost $17 \%$ of Biharis are in either the Schedule Castes or Schedule Tribal communities ( $15.7 \%$ and $0.9 \%$ respectively). ${ }^{18}$ These groups are granted a "special status" under the Indian Constitution, but they continue to differ systematically from other social groups. ${ }^{18}$ Scheduled Castes and Tribes have the lowest mean body mass index (BMI) values compared with General and Other Castes, and $64.7 \%$ of Scheduled Castes and $57.5 \%$ of scheduled Tribes experience chronic energy deficiency (CED).${ }^{18}$ Also, the higher castes in Bihar have control of most of the natural resources and government jobs. ${ }^{21}$

\section{Ethical considerations for clinical trials on L. donovani in Bihar Collaborative partnership}

The design and conduct of a clinical trial should be a collaborative partnership between sponsoring and host countries. Community researchers, health policy makers, and community members should be involved in a meaningful way throughout the research process. ${ }^{10}$ It is advisable that any international clinical trial conducted in Bihar include participation and support from the Rajendra Memorial Research Institute of Medical Sciences (RMRI). The RMRI is a branch of the Indian Council of Medical Research (ICMR) and is located in the city of Patna, Bihar, India. ${ }^{26}$ The institute's primary focus is visceral leishmaniasis, which is known as kala-azar in the region. RMRI research on visceral leishmaniasis includes epidemiological studies, clinical studies and community projects. Such a partnership would include the fair distribution of financial, intellectual, and other benefits that result from the study.

\section{Social value}

There is clearly social value in discovering a new treatment that is effective and well tolerated among Biharis. To ensure social value, a plan should be established to translate research findings into healthcare delivery for the host communities. This plan requires capacity building in host communities that may include training medical staff and community health workers, and providing equipment and additional resources. ${ }^{10}$

\section{Fair selection of study population}

The study population should be selected to ensure both scientific validity and fair distribution of the risks and benefits of the research. Visceral leishmaniasis disproportionally 
affects children and men in Bihar, and these groups should be targeted for clinical trials in the region. The disease also disproportionally affects low-income Biharis, where one hospital-based study found that $30 \%$ of cases were among those living below the poverty line (BPL) (annual income $<$ Rs 3312), and $18 \%$ of cases were among those at the national average or above ( $>$ Rs 9600). ${ }^{25}$ Children and impoverished persons are vulnerable groups, and researchers should establish safeguards to prevent exploitation of these populations. Targeting vulnerable populations is only justifiable if they are likely to benefit from their participation. It is unethical in terms of justice for a clinical trial to result in improved treatments only for those other than the study population. For example, it violates the principle behind fair selection of study population if a trial that resulted in improved treatment was comprised mainly of adult males BPL and the drug therapy was financially inaccessible to this group.

\section{Independent review}

The study proposal and protocol should be approved by an appropriate, local review board and meet the ethical standards established by the ICMR. The ICMR states requirements for the ethical conduct of human research in the Ethical Guidelines for Medical Research on Human Participants and has ethical standards similar to those in the US. It may also be appropriate to submit the study to a US institutional review board (IRB), though it is not ethically required to do so, but may be mandated for regulatory reasons. Federally funded studies are required to have US IRB approval, although it is more ethically relevant to strengthen the existing independent review procedures in India. It is possible that a study can be approved by an ethics review board in the US and still have ethical shortcomings, particularly with regards to the ethical principles developed by Emanuel et al for research in developing countries..$^{10}$ The issue of informed consent is an appropriate example of this. A US IRB may require Bihari study participants to provide voluntary informed consent. However, obtaining meaningful consent may be difficult because of deference to medical professionals and the strong hierarchal forces of the caste system.

\section{Informed consent}

The informed consent process should be designed with members of the community who will serve as the study population. This approach will help ensure the appropriateness of the ICF, including the language style (language level, wording, etc) and possible compensation. Compensation may be expected in some communities, but interpreted as offensive in others. ${ }^{10}$ Community members and key stakeholders in the region can provide information regarding what level of compensation is socially and economically appropriate. Due to a largely illiterate population, the ICF should be phrased appropriately and read aloud to the potential participants and a thumb print can substitute for a signature.

\section{Ancillary care}

Medical and other needs will likely be identified during the selection of study participants. The level of responsibility of the researchers to provide care that goes beyond the requirements of scientific validity and participant compensation are disputed among researchers and bioethicists. ${ }^{27}$ It has been difficult for bioethicists to develop systematic standards for ancillary care, partly because ancillary care responsibilities are highly contextual and depend on the nature of the researcherparticipant relationship. ${ }^{27}$ The level of obligation increases as the researcher-participant relationship is strengthened, such as through multiple, lengthy interactions ${ }^{27}$ Researchers should anticipate medical needs common to their population and develop a strategy in advance for addressing these needs within their sample. ${ }^{27}$ Study budgets may need to be adjusted to accommodate predictable ancillary care needs. ${ }^{27}$

\section{Clinical trial example}

The 1999-2000 phase 3 clinical trial comparing oral miltefosine and intravenous amphotericin B provides a scientifically sound description of the study procedures and results and a helpful comparison of oral miltefosine to existing treatments. ${ }^{28}$ The trial took place at 3 medical centers in Bihar, and the study protocol, consent forms, and other documents were approved by an ethics committee at each site. ${ }^{28}$ Eligible patients were at least 12 years old and met the stated inclusion/exclusion criteria. ${ }^{28}$ However, more information about the population is needed to assess the study with regards to the ethical considerations discussed, particularly social value to the host communities and ancillary care. The social value of an effective and well tolerated drug is clear, but only if the new treatment is accessible to the study population and host communities. A plan describing healthcare delivery of the new drug would be beneficial in evaluating the social value criterion. Ancillary care was not discussed in the document, and it is unclear what happened to those exempted by the exclusion criteria. Individuals were excluded due to the presence of major medical illness, including human immunodeficiency virus and severe malaria; or platelet, white-cell, hemoglobin, bilirubin, 
and/or blood urea abnormalities. ${ }^{28}$ Also, a discussion of treatment costs and plans for translating research findings into healthcare delivery is needed in all cases where the study population is burdened by extreme poverty and/or when the healthcare infrastructure is poor.

The primary job of clinical trial publications is to inform the scientific community and general public of the benefits, complications, and other relevant issues of new treatments. The need to meet certain established ethical guidelines is well understood. Publications on research involving human participants usually state whether or not the study has been approved by an ethics committee as well as a brief description of the study population and inclusion/exclusion. However, disclosure of pertinent ethical considerations is needed in certain cases, including international clinical trials taking place in developing countries. Doing so would facilitate open discussion of contemporary ethical issues researchers experience in clinical trials and lead to improvements in addressing these issues.

\section{Conclusion}

The great disease burden in Bihar caused by L. Donovani necessitates the need for clinical trials in the region. The procedures used to apply universal ethical principles are necessarily context specific and depend on many social, cultural, and political factors. ${ }^{15}$ Scientists involved in international research must be knowledgeable of and sensitive to the social, economic, and political issues relevant to their participants and the ways in which these factors contribute to their health status. ${ }^{16}$ The description of the Bihar context and relevant ethical considerations provided here should facilitate the design and conduct of ethical clinical trials for L. donovani in the Bihar region of India. Community researchers, health policy makers and community members should be involved in a meaningful way throughout the research process. ${ }^{10}$ Clinical research should strive to provide social value, such as educating participants about ways to prevent transmission of leishmaniasis. It is important that participants are selected fairly and that those most affected by the disease will be able to participate. It is an issue of social justice that the most poor have the highest rates of leishmaniasis. Steps should be taken to ensure their inclusion in the study and post-trial access to treatments. Perhaps the most challenging task for a Western researcher is to ascertain if true informed consent is obtained, and this task will be made possible with the help of key community personnel to guide the informed consent process. Because ethical research is context specific, the context and applicable ethical principles presented in this paper may provide insight to Western researchers who wish to conduct clinical research in Bihar.

\section{Disclosure}

The authors report no conflict of interest for this paper.

\section{References}

1. Chappuis F, Sundar S, Hailu A, et al. Visceral leishmaniasis: what are the needs for diagnosis, treatment and control? Nature. 2007;5:873-882.

2. Gramiccia M, Gradoni L. The current status of zoonotic leishmaniases and approaches to disease control. Int J Parasitol. 2005;35:1169-1180.

3. Hailu A, Gramiccia M, Kager PA. Viseral leishmaniasis in Aba-Roba, south-western Ethopia: prevalence and incidence of active and subclinical infections. Ann Trop Med Parasit. 2009;103:659-670.

4. World Health Organization. Leishmania and HIV in Gridlock. Geneva: World Health Organization; 1998:1-28.

5. Sundar S. Drug resistance in Indian visceral leishmaniasis. Trop Med Int Health. 2001;6:849-854.

6. Desjeux P. Leishmaniasis: current situation and new perspectives. Comp Immunol Microb. 2004;27:305-318.

7. National Institutes of Health. Protecting Human Research Participants. DC; 2008.

8. Council for International Organizations of Medical Sciences. International Ethical Guidelines for Biomedical Research Involving Human Subjects. Geneva, Switzerland; 2002.

9. Shapiro HT, Meslin EM. Ethical issues in the design and conduct of clinical trials in developing countries. New Engl J Med. 2001;345(2): 139-142.

10. Emanuel EJ, Wendler D, Killen J, Grady C. What makes clinical research in developing countries ethical? The benchmarks of ethical research. J Infect Dis. 2004;189:930-937.

11. Wma.net [homepage on the Internet]. World Medical Association Declaration of Helsinki. Available from: http://www.wma.net/ en/30publications/10policies/b3/index.html. Accessed Feb 20, 2010.

12. SocialMedicine.org [homepage on the Internet]. Social Medicine Portal. Available from: http://www.socialmedicine.org/2008/06/01/ethics/ fda-abandons-declaration-of-helsinki-for-international-clinical-trials/. Accessed Feb 23, 2010.

13. Flory J, Emanuel E. Interventions to improve research participants' understanding in informed consent for research: a systematic review. J Amer Med Assoc. 2004;292(13):1593-1601.

14. Indian Council of Medical Research. Ethical Guidelines for Biomedical Research on Human Participants. New Delhi; 2006.

15. Tangwa GB. Between universalism and relativism: a conceptual exploration of problems in formulating and applying international biomedical ethical guidelines. J Med Ethics. 2004;30:63-67.

16. Benatar SR, Singer PA. A new look at international research ethics. Brit Med J. 2000;321:824-826.

17. Thakur CP. Socio-economics of visceral leishmaniasis in Bihar (India). T Roy Soc Trop Med H. 2000;94:156-157.

18. Chakrabarty S, Pal M, Bharati S, Bharati P. Body form and nutritional status among adult males of different social groups in Orissa and Bihar States in India. Homo. 2008;59:235-251.

19. Cerf BJ, Jones TC, Badaro R, Sampaio D, Teixeira R, Johnson WD Jr. Malnutrition as a risk factor for severe visceral leishmaniasis. J Infect Dis. 1987;156:1030-1033.

20. Sharma NL, Mahajan VK, Negi AK, Verma GK. The rK39 immunochromatic dipstick testing: a study for K39 seroprevalence in dogs and human leishmaniasis patients for possible animal reservoir of cutaneous and visceral leishmaniasis in endemic focus of Satluj river valley of Himachal Pradesh (India). Indian J Dermatol VE. 2009;75:52-55.

21. Parker B, Kozel V. Understanding poverty and vulnerability in India's Uttar Pradesh and Bihar: a Q-squared approach. World Dev. 2007;35: 296-311.

22. Sundar S. Drug resistance in Indian visceral leishmaniasis. Trop Med Int Health. 2001;6:849-854. 
23. Sundar S, Chakravarty J, Agarwal D, Rai M, Murray HW. Single-dose liposomal amphotericin B for visceral leishmaniasis in India. NEJM. 2010;362:504-512.

24. Rao KS. Financing of Health in India. Delhi: Institute of Economic Growth, University of Delhi Enclave; 2005:239-255.

25. Sinha PK, Ranjan A, Singh VP, et al. Visceral leishmaniasis (kala-azar) the Bihar (India) perspective. J Infection. 2006;53:60-64.
26. Rmrims.org.in [homepage on the Internet]. Bihar: Rajendra Memorial Research Institute of Medical Sciences. Available from www.rmrims. org.in. Accessed Feb 22, 2010.

27. Dickert N, Wendler D. Ancillary care obligation of medical researchers. JAMA-J Am Med Assoc. 2009;307(4):424-428.

28. Sundar S, Jha TK, Thakur CP, et al. Oral miltefosine for Indian visceral leishmaniasis. New Engl J Med. 2002:347(22):1739-1746.

Open Access Journal of Clinical Trials

\section{Publish your work in this journal}

The Open Access Journal of Clinical Trials is an international, peerreviewed, open access journal publishing original research, reports, editorials, reviews and commentaries on all aspects of clinical tria design, management, legal, ethical and regulatory issues, case record form design, data collection, quality assurance and data auditing methodologies. The manuscript management system is completely online and includes a very quick and fair peer-review system, which is all easy to use. Visit http://www.dovepress.com/testimonials.php to read real quotes from published authors.

Submit your manuscript here: http://www.dovepress.com/open-access-journal-of-clinical-trials-journal 